\title{
EXACT REPRESENTATIONS FOR TUMOUR INCIDENCE FOR SOME DENSITY-DEPENDENT MODELS
}

\author{
P. R. PARTHASARATHY AND KLAUS DIETZ
}

Received 10 March 2005

Carcinogenesis is a multistage random process involving generic changes and stochastic proliferation and differentiation of normal cells and genetically altered stem cells. In this paper, we present the probability of time to tumour onset for a carcinogenesis model wherein the cells grow according to a birth and death process with density-dependent birth and death rates. This is achieved by transforming the underlying system of difference equations which results in a continued fraction. This continued fraction approach helps us to find the complete solutions. The popular Moolgavkar-Venzon-Knudson (MVK) model assumes constant birth, death, and transition rates.

\section{Introduction}

Cancer arises from the stepwise accumulation of genetic changes that confer upon an incipient neoplastic cell the properties of unlimited, self-sufficient growth and resistance to normal homeostatic regulatory mechanisms. Advances in human genetics and molecular and cellular biology have identified a collection of cell phenotypes that are required for malignant transformation. Alterations to the DNA inside cells can endow cells with morbid "superpowers," such as the ability to grow anywhere and to continue dividing indefinitely. A long held theory focusses on mutations to a relatively small set of cancerrelated genes as the decisive events in the transformation of healthy cells to malignant tumours. Recently, however, other theories have emerged to challenge this view (Gibbs [6]).

It is now universally recognized that carcinogenesis is a multistage random process involving genetic changes and stochastic proliferation and differentiation of normal stem cells and genetically altered stem cells (Tan [16]). Among a multitude of carcinogenesis models, the Moolgavkar-Venzon-Knudson (MVK) model seems to have attracted most of the research efforts and is enjoying wide applicability in both epidemiological and animal experimental studies (Moolgavkar [11]).

Under the MVK model, a malignant cancer cell is assumed to arise following the occurrence of two critical mutations in a normal stem cell. Initiated cells that have sustained the first mutation undergo a birth and death process (details about applications of birth 
and death processes can be attained from Parthasarathy and Lenin [15]). If the birth rate exceeds the death rate, this results in a clonal expansion of initiated cells. The number of stem cells at the risk of transformation is allowed to increase in a deterministic fashion to reflect tissue growth and development. A detailed review of biological, mathematical, and statistical aspects of the two-stage model has been provided by Moolgavkar and Luebeck in [12]. The mathematical properties of the two-stage model are also described in (Tan [16]). For this basic two-stage (MVK) model, an assumption that a single malignant cell is equivalent to a tumour is made in order to simplify mathematics. Exact expression for the probability generating function for the two-stage model is derived by Denes and Krewski [5] in terms of hypergeometric functions. They point out that this exact and approximate expression of Moolgavkar and Venzon in [13] exhibits qualitative differences.

In the two-stage model of carcinogenesis, the intermediate cells are assumed to grow as a linear birth and death process. It is important to point out that even though they all constitute forms of cancer, there is a quantitative difference between the formation and growth of various solid tumuors and disseminated cancers such as leukemia (Afenya and Calderón[1]). Further, the process of carcinogenesis is significantly influenced by environmental factors underlying the individual.

In this paper, we assume that the initiated cells grow as a birth and death process with density-dependent birth and death rates. Research into the time to tumour onset has occupied a central place in carcinogenesis modelling, because it bridges the gap between the theory and application of models. The predominant method of computing these quantities is an application of the characteristic method of solving first-order partial differential equations.

We obtain exact expressions for the time to tumour onset for several cases of densitydependent birth and death rates. This is achieved by transforming the underlying system of differential equations using Laplace transform to a system of difference equations which results in a continued fraction. This continued fraction helps us to find the complete solutions.

\section{A density-dependent carcinogenesis model}

To develop stochastic models of carcinogenesis, the traditional approach is by way of Markov theories. The basic approach along this line consists of four basic steps:

(1) derive the probability generating function of the number of tumours,

(2) derive the incidence function of tumours,

(3) derive the probability distribution of time to tumour onset, and

(4) obtain the probabilities of the number of tumours.

The problem with such a modelling approach is that if the model is more complex than the framework of the two-stage model, the modelling process becomes too complicated to be useful. For the basic two-stage (MVK) model, an assumption that a single malignant cell is equivalent to a tumour must be made in order to simplify mathematics.

First we consider a modified MVK model. Assume that there are two compartments, intermediate cell compartment and tumour cell compartment and let $X_{1}(t)$ and $X_{2}(t)$ denote the number of cells in each compartment. 
If $X_{1}(t)=n$, birth, death, and mutation rates are $\lambda, \mu$, and $\nu$ (i.e., $n(\lambda / n), n(\mu / n)$, and $n(\nu / n))$, respectively. Thus these rates decrease as $n$ increases, thereby regulating the cell population. That is, $\lambda / n, \mu / n$, and $\nu / n$ are the birth, death, and mutation rates, when $n$ cells are present. (In MVK model these rates are $n \lambda, n \mu$, and $n \nu$.)

Let $G\left(s_{1}, s_{2} ; t\right)=\sum \sum p_{m n}(t) s_{1}^{m} s_{2}^{n}$ denote the probability generating function of $\left(X_{1}(t)\right.$, $\left.X_{2}(t)\right)$. Let $P_{m n}(t)=P\left(X_{1}(t)=m, X_{2}(t)=n\right)$.

By random variable technique (see Bailey [2]),

$$
\frac{\partial G}{\partial t}=\left[\lambda\left(s_{1}-1\right)+\mu\left(\frac{1}{s_{1}}-1\right)+\nu\left(s_{2}-1\right)\right]\left[G-g_{0}\left(s_{2}, t\right)\right]
$$

where $g_{m}\left(s_{2}, t\right)=\sum_{n=0}^{\infty} P\left(X_{1}(t)=m, X_{2}(t)=n\right) s_{2}^{n}$. Following convention, it is assumed that at time $t=0$ only the first compartment is nonempty, and hence the initial condition is $P\left(X_{1}(0)=N, X_{2}(0)=0\right)=1$, that is, $G\left(s_{1}, s_{2} ; 0\right)=s_{1}^{N}$.

Note that $p_{0 m}^{\prime}=\mu p_{1 m}$, for $m=0,1,2, \ldots$ and hence

$$
g_{0}^{\prime}\left(s_{2}, t\right)=\mu g_{1}\left(s_{2}, t\right)
$$

If $P\left(s_{1}, s_{2} ; t\right)=G\left(s_{1}, s_{2} ; t\right)-g_{0}\left(s_{2}, t\right)$, then

$$
\frac{\partial P}{\partial t}=\left[\lambda\left(s_{1}-1\right)+\mu\left(\frac{1}{s_{1}}-1\right)+\nu\left(s_{2}-1\right)\right] P-\mu g_{1}\left(s_{2}, t\right) .
$$

Solving this differential equation,

$$
\begin{aligned}
P\left(s_{1}, s_{2} ; t\right)= & s_{1}^{N} e^{\left[\lambda\left(s_{1}-1\right)+\mu\left(1 / s_{1}-1\right)+\nu\left(s_{2}-1\right)\right] t} \\
& -\mu \int_{0}^{t} g_{1}\left(s_{2}, y\right) e^{\left[\lambda\left(s_{1}-1\right)+\mu\left(1 / s_{1}-1\right)+\nu\left(s_{2}-1\right)\right](t-y)} d y .
\end{aligned}
$$

We use the fact that the generating function of modified Bessel functions is given by

$$
\sum_{n=-\infty}^{\infty} I_{n}(\alpha t)(\beta s)^{n}=e^{(\lambda s+\mu / s) t}
$$

where $\alpha=2 \sqrt{\lambda \mu}$ and $\beta=\sqrt{\lambda / \mu}$.

Thus,

$$
\begin{aligned}
P\left(s_{1}, s_{2} ; t\right)= & s_{1}^{N} e^{-(\lambda+\mu+\nu) t} \sum_{n=-\infty}^{\infty} I_{n}(\alpha t)\left(\beta s_{1}\right)^{n} e^{\nu s_{2} t} \\
& -\mu \int_{0}^{t} g_{1}\left(s_{2}, y\right) e^{-(\lambda+\mu+\gamma)(t-y)} \sum_{n=-\infty}^{\infty} I_{n}(\alpha(t-y))\left(\beta s_{1}\right)^{n} e^{\nu s_{2}(t-y)} d y .
\end{aligned}
$$

Comparing the coefficient of $s_{1}^{m}$ on both sides, we obtain

$$
\begin{aligned}
g_{m}\left(s_{2} ; t\right)= & e^{-(\lambda+\mu+\nu) t} I_{m-N} \beta^{m-N} e^{\nu s_{2} t} \\
& -\mu \int_{0}^{t} g_{1}\left(s_{2} ; y\right) e^{-(\lambda+\mu+\nu)(t-y)} I_{m}(\alpha(t-y)) \beta^{m} e^{\nu s_{2}(t-y)} d y .
\end{aligned}
$$


2658 Density-dependent carcinogenesis models

Comparing the coefficient of $s_{1}^{-m}$ on both sides, we obtain

$$
\begin{aligned}
0= & e^{-(\lambda+\mu+\nu) t} I_{-(m-N)} \beta^{-(m-N)} e^{\nu s_{2} t} \\
& -\mu \int_{0}^{t} g_{1}\left(s_{2} ; y\right) e^{-(\lambda+\mu+\nu)(t-y)} I_{-m}(\alpha(t-y)) \beta^{-m} e^{\nu s_{2}(t-y)} d y .
\end{aligned}
$$

Multiplying (2.7) by $\beta^{-m}$ and (2.8) by $\beta^{m}$ and subtracting, we get

$$
g_{m}\left(s_{2}, t\right)=e^{-(\lambda+\mu+\nu) t} \frac{I_{m-N}(\alpha t)-I_{m+N}(\alpha t)}{\beta^{N-m}} e^{\nu s_{2} t} \quad \text { for } m=1,2, \ldots
$$

using $I_{-m}=I_{m}$.

But $g_{0}^{\prime}\left(s_{2}, t\right)=\mu g_{1}\left(s_{2}, t\right)$ and $g_{0}\left(s_{2}, 0\right)=0$ :

$$
g_{0}\left(s_{2}, t\right)=\mu \int_{0}^{t} g_{1}\left(s_{2}, u\right) d u=\mu \int_{0}^{t} e^{-(\lambda+\mu+\nu) y} \frac{I_{1-N}(\alpha y)-I_{1+N}(\alpha y)}{\beta^{N-1}} e^{\nu s_{2} y} d y .
$$

Let $T$ be the time that a cancer tumour develops for the first time. Let $f(t)$ be the probability density function of $T$ and $F(t)$ the cumulative distribution function of $T$. If $S(t)=$ $1-F(t)$, the incidence function $h(t)$ of tumour onset at time $t$ is defined by $h(t)=$ $-d \ln S(t) / d t$ :

$$
P(T>t)=G(1,0 ; t)=P(1,0 ; t)+g_{0}(0, t)
$$

From (2.4)

$$
P(1,0 ; t)=e^{-v t}-\mu \int_{0}^{t} g_{1}(0, y) e^{-v(t-y)} d y
$$

and from (2.10)

$$
g_{0}(0, t)=\mu \int_{0}^{t} e^{-(\lambda+\mu+\nu) y} \frac{I_{1-N}(\alpha y)-I_{1+N}(\alpha y)}{\beta^{N-1}} d y
$$

Thus,

$$
\begin{aligned}
P(T>t)= & e^{-v t}-\mu \int_{0}^{t} e^{-(\lambda+\mu+\nu) y} \frac{I_{1-N}(\alpha y)-I_{1+N}(\alpha y)}{\beta^{N-1}} e^{\nu(t-y)} d y \\
& +\mu \int_{0}^{t} e^{-(\lambda+\mu+\nu) y} \frac{I_{1-N}(\alpha y)-I_{1+N}(\alpha y)}{\beta^{N-1}} d y \\
= & e^{-v t}+\mu \int_{0}^{t}\left[1-e^{-\nu(t-y)}\right] e^{-(\lambda+\mu+\nu) y} \frac{I_{1-N}(\alpha y)-I_{1+N}(\alpha y)}{\beta^{N-1}} d y \\
= & e^{-v t}+\mu \int_{0}^{t}\left[1-e^{-v(t-y)}\right] e^{-(\lambda+\mu+\nu) y} \frac{2 N I_{N}(\alpha y)}{\alpha y \beta^{N-1}} d y, \\
P(T>t) \longrightarrow & \frac{(2 \mu)^{N}}{\left[\lambda+\mu+\nu+\sqrt{(\lambda+\mu+\nu)^{2}-4 \lambda \mu}\right]^{N}} \text { as } t \longrightarrow \infty .
\end{aligned}
$$


After considerable simplification,

$$
\begin{aligned}
G\left(s_{1}, s_{2} ; t\right)= & s_{1}^{N} e^{\left[\lambda\left(s_{1}-1\right)+\mu\left(1 / s_{1}-1\right)+\nu\left(s_{2}-1\right)\right] t} \\
& +\mu \int_{0}^{t}\left\{1-e^{\left[\lambda\left(s_{1}-1\right)+\mu\left(1 / s_{1}-1\right)+\nu\left(s_{2}-1\right)\right](t-y)}\right\} g_{1}\left(s_{2}, y\right) d y .
\end{aligned}
$$

From this, if $s_{2}=1$,

$$
\begin{aligned}
G_{1}\left(s_{1}, t\right)= & \sum P\left(X_{1}(t)=m\right) s_{1}^{m}=s_{1}^{N} e^{\left[\lambda\left(s_{1}-1\right)+\mu\left(1 / s_{1}-1\right)\right] t} \\
& +\mu \int_{0}^{t}\left\{1-e^{\left[\lambda\left(s_{1}-1\right)+\mu\left(1 / s_{1}-1\right)\right](t-y)}\right\} g_{1}(1, y) d y
\end{aligned}
$$

and if $s_{1}=1$,

$$
G_{2}\left(s_{2}, t\right)=\sum P\left(X_{2}(t)=n\right) s_{2}^{n}=e^{\nu\left(s_{2}-1\right) t}+\mu \int_{0}^{t}\left\{1-e^{\nu\left(s_{2}-1\right)(t-y)}\right\} g_{1}\left(s_{2}, y\right) d y .
$$

Therefore,

$$
\begin{gathered}
m_{1}(t)=E\left(X_{1}(t)\right)=N+(\lambda-\mu) t-(\lambda-\mu) \int_{0}^{t} g_{0}(1, y) d y, \\
E\left(X_{1}(t)\left(X_{1}(t)-1\right)\right)=N(N-1)+2 \mu \int_{0}^{t}\left[1-g_{0}(1, y)\right] d y+2(\lambda-\mu) E\left(X_{1}(t)\right), \\
m_{2}(t)=E\left(X_{2}(t)\right)=v \int_{0}^{t}\left[1-g_{0}(1, y)\right] d y \\
E\left(X_{2}(t)\left(X_{2}(t)-1\right)\right)=2 v \int_{0}^{t}\left[m_{2}(y)-\mu g_{1}(1, y)\right] d y \\
E\left(X_{1}(t) X_{2}(t)\right)=(\lambda-\mu) \int_{0}^{t}\left[m_{2}(y)-\mu g_{1}(1, y)\right] d y+v \int_{0}^{t} m_{1}(y) d y .
\end{gathered}
$$

Note that

$$
g_{1}(1, t)=e^{-(\lambda+\mu) t} \frac{I_{N-1}(\alpha t)-I_{N+1}(\alpha t)}{\beta^{N-1}}, \quad g_{0}(1, t)=\mu \int_{0}^{t} g_{1}(1, y) d y .
$$

These give means and variances of $X_{1}(t), X_{2}(t)$ and covariance between $X_{1}(t)$ and $X_{2}(t)$.

In the above analysis we have used generating functions. However, this technique may not be available for other density-dependent rates. We use instead the continued fraction approach. Perhaps, this is the first attempt in this direction. This approach leads to an explicit expression for the Laplace transform of the time to tumour onset. Given a set of birth and death parameters, one can invert this transform numerically. However, for certain birth and death rates, this Laplace transform can lead to closed form expressions for the time to tumour onset as illustrated in Section 4. 


\section{Exact representation}

Consider a model of carcinogenesis consisting of intermediate and tumour cells.

An intermediate cell, when the system size at time $t$ is $n$, produces two intermediate cells with probability $\lambda_{n} h+o(h)$ during $(t, t+h)$, dies with probability $\mu_{n} h+o(h)$ or produces an intermediate cell and a tumour cell with probability $v_{n} h+o(h)$. We assume that intermediate cells are generated from normal ones by a Poisson process and this rate can be accommodated with rate, $\lambda_{n}$.

Let $X_{1}(t)$ and $X_{2}(t)$ denote the number of intermediate and tumour cells at time $t$. Let $P_{i j}(t)=P\left(X_{1}(t)=i, X_{2}(t)=j\right)$.

These transition probabilities satisfy Kolmogorov forward differential equations:

$$
\begin{gathered}
P_{m 0}^{\prime}(t)=\lambda_{m-1} P_{m-10}(t)+\mu_{m+1} P_{m+10}(t) \\
-\left(\lambda_{m}+\mu_{m}+\nu_{m}\right) P_{m 0}(t), \quad m=2,3, \ldots, \\
P_{00}^{\prime}(t)=\mu_{1} P_{10}(t), \\
P_{10}^{\prime}(t)=\mu_{2} P_{20}(t)-\left(\lambda_{1}+\mu_{1}+\nu_{1}\right) P_{10}(t) .
\end{gathered}
$$

For $m=1,2,3, \ldots$ and $n=1,2,3, \ldots$

$$
\begin{gathered}
P_{m n}^{\prime}(t)=\lambda_{m-1} P_{m-1 n}(t)+\mu_{m+1} P_{m+1 n}(t)+\nu_{m} P_{m n-1}(t) \\
-\left(\lambda_{m}+\mu_{m}+\nu_{m}\right) P_{m n}(t), \\
P_{0 n}^{\prime}(t)=\mu_{1} P_{1 n}(t) .
\end{gathered}
$$

Assume $P_{10}(0)=1$. The probability of time to tumour onset, starting with one initiated cell is given by

$$
q_{0}(t)=P(T>t)=\sum_{i=0}^{\infty} P_{i 0}=\sum_{i=0}^{\infty} P\left(X_{1}(t)=i, X_{2}(t)=0\right) .
$$

Let $f_{m n}(z)=\int_{0}^{\infty} e^{-z t} P_{m n}(t) d t$ be the Laplace transform of $P_{m n}(t)$. The transition probability $P_{10}(t)$ plays a vital role in the study of the incidence function. We show that $f_{10}(z)$ can be expressed as a continued fraction.

From (3.1)

$$
\begin{gathered}
z f_{00}(z)=\mu_{1} f_{10}(z), \\
z f_{10}(z)-1=\mu_{2} f_{20}(z)-\left(\lambda_{1}+\mu_{1}+\nu_{1}\right) f_{10}(z), \\
z f_{m 0}(z)=\lambda_{m-1} f_{m-10}(z)+\mu_{m+1} f_{m+10}(z)-\left(\lambda_{m}+\mu_{m}+\nu_{m}\right) f_{m 0}(z) .
\end{gathered}
$$

From (3.5)

$$
\left(z+\lambda_{1}+\mu_{1}+v_{1}\right) f_{10}(z)=1+\mu_{2} f_{20}(z)
$$

or

$$
\left(z+\lambda_{1}+\mu_{1}+\nu_{1}\right)-\mu_{2} \frac{f_{20}(z)}{f_{10}(z)}=\frac{1}{f_{10}(z)}
$$


or

$$
f_{10}(z)=\frac{1}{z+\lambda_{1}+\mu_{1}+\nu_{1}-\mu_{2} \frac{f_{20}(z)}{f_{10}(z)}}
$$

From (3.6), for $m=2,3, \ldots$

$$
\left(z+\lambda_{m}+\mu_{m}+v_{m}\right)-\mu_{m+1} \frac{f_{m+10}(z)}{f_{m 0}(z)}=\lambda_{m-1} \frac{f_{m-10}(z)}{f_{m 0}(z)}
$$

or

$$
\frac{f_{m 0}(z)}{f_{m-10}(z)}=\frac{\lambda_{m-1}}{\left(z+\lambda_{m}+\mu_{m}+\nu_{m}\right)-\mu_{m+1} \frac{f_{m+10}(z)}{f_{m 0}(z)}}
$$

Thus

$$
f_{10}(z)=\frac{1}{z+\lambda_{1}+\mu_{1}+\nu_{1}-\frac{\lambda_{1} \mu_{2}}{z+\lambda_{2}+\mu_{2}+\nu_{2}-\mu_{3} \frac{f_{30}}{f_{20}}}}
$$

Continuing in this way,

$$
f_{10}(z)=\frac{1}{z+\lambda_{1}+\mu_{1}+\nu_{1}-\frac{\lambda_{1} \mu_{2}}{z+\lambda_{2}+\mu_{2}+\nu_{2}-\frac{\lambda_{2} \mu_{3}}{z+\lambda_{3}+\mu_{3}+\nu_{3}-\cdots}}}
$$

Assume $v_{i}=v$, for all $i$. Then the continued fraction expansion form of $f_{10}(z)$ can be written in a convenient form,

$$
f_{10}(z)=\frac{1}{z+\lambda_{1}+\mu_{1}+\nu}-\frac{\lambda_{1} \mu_{2}}{z+\lambda_{2}+\mu_{2}+\nu-} \frac{\lambda_{2} \mu_{3}}{z+\lambda_{3}+\mu_{3}+\nu-} \cdots
$$

By inverting this Laplace transform, $P_{10}(t)$ can be obtained:

$$
P_{10}(t)=e^{-v t} L^{-1}\left(\frac{1}{z+\lambda_{1}+\mu_{1}-} \frac{\lambda_{1} \mu_{2}}{z+\lambda_{2}+\mu_{2}-} \frac{\lambda_{2} \mu_{3}}{z+\lambda_{3}+\mu_{3}-} \cdots\right)=e^{-v t} L^{-1}(R(z)) .
$$


Here

$$
R(z)=\frac{1}{z+\lambda_{1}+\mu_{1}-} \frac{\lambda_{1} \mu_{2}}{z+\lambda_{2}+\mu_{2}-} \frac{\lambda_{2} \mu_{3}}{z+\lambda_{3}+\mu_{3}-} \cdots
$$

CF approximations occupy a remarkable place in mathematical literature due to their interesting convergence properties and also due to their connections with many branches of mathematics like number theory, special functions, differential equations, moment problems, orthogonal polynomials, and so forth (Lorentzen and Waadeland [8]). Approximations employing CFs often provide a good representation for transcendental functions. They are generally much more valid than the classical representation by power series. On account of their algorithmic nature, they are used in numerical analysis, computer science, automata, electronic communication, and so forth. Their importance has grown further with the advent of fast computing facilities. A systematic study of the theory of CFs with stress on computations can be found in [7]. Its application to the study of BDPs was initiated by Murphy and O'Donohoe [14].

Again, from (3.1)

$$
q_{0}^{\prime}(t)=\sum_{m=0}^{\infty} P_{m 0}^{\prime}(t)=-\left(\nu_{1} P_{10}+v_{2} P_{20}+\cdots\right) .
$$

We assume that $v_{n}=v$, independent of $n$. This is valid as the probability of getting a tumour cell is extremely small:

$$
q_{0}^{\prime}(t)=-v\left(P_{10}+P_{20}+\cdots\right)=-v\left(q_{0}(t)-P_{00}(t)\right) .
$$

Since initially there is no cancer cell, $q_{0}(0)=1$,

$$
\begin{aligned}
\left(q_{0}(t) e^{\nu t}\right)^{\prime} & =v e^{\nu t} P_{00}(t) \\
q_{0}(t) e^{\nu t} & =1+v \int_{0}^{t} e^{\nu y} P_{00}(y) d y \\
q_{0}(t) & =e^{-v t}+v \int_{0}^{t} e^{-v(t-y)} P_{00}(y) d y .
\end{aligned}
$$

Using (3.1), we express $q_{0}(t)$ in terms of $P_{10}(t)$ for future use:

$$
P_{00}^{\prime}(t)=\mu_{1} P_{10}(t), \quad P_{00}(t)=\mu_{1} \int_{0}^{t} P_{10}(y) d y .
$$

Substituting this in (3.19) and changing the order of integration,

$$
q_{0}(t)=e^{-v t}+\mu_{1} \int_{0}^{t} P_{10}(v)\left(1-e^{-v(t-v)}\right) d v
$$


The hazard rate is given by $q_{0}^{\prime}(t) / q_{0}(t)$. Taking Laplace transform on both sides of (3.19), we obtain

$$
\begin{gathered}
\hat{q}_{0}(z)=\frac{1}{v+z}+\mu_{1} \frac{v}{z(z+v)} P_{10}(z), \\
\lim _{t \rightarrow \infty} q_{0}(t)=\lim _{z \rightarrow 0} z \hat{q}_{0}(z)=\mu_{1} f_{10}(0)=\mu_{1} R(v) .
\end{gathered}
$$

We will now give several examples wherein explicit expressions for $q_{0}(t)$ can be obtained. This is achieved by identifying certain continued fractions associated with Laplace transform, $R(z)$ of $e^{\nu t} P_{10}(t)$.

\section{Examples}

Example 4.1 (modified MVK model). Here $\lambda_{n}=n \lambda, \mu_{n}=n \mu$ whereas $\nu_{n}=\nu$ (for MVK model $\left.v_{n}=n v\right)$ :

$$
\begin{aligned}
R(z) & =\frac{1}{z+\lambda+\mu-} \frac{1 \cdot 2 \lambda \mu}{z+2 \lambda+2 \mu-} \frac{2 \cdot 3 \lambda \mu}{z+3 \lambda+3 \mu-} \cdots \\
& =\frac{1 / \lambda}{\frac{z+\lambda+\mu}{\lambda}-\frac{1 \cdot 2(\mu / \lambda)}{\frac{z+2 \lambda+2 \mu}{\lambda}-} \frac{2 \cdot 3(\mu / \lambda)}{\frac{z+3 \lambda+3 \mu}{\lambda}-} \cdots} \\
R(z) & =\frac{1 / \lambda}{u+c+1-} \frac{1 \cdot 2 c}{u+2+2 c-} \frac{2 \cdot 3 c}{u+3+3 c-} \cdots \quad\left(c=\frac{\mu}{\lambda}, u=\frac{z}{\lambda}\right), \\
& =\frac{1 / \lambda}{v+2-} \frac{1 \cdot 2 c}{v+3+c-} \frac{2 \cdot 3 c}{z+2 c+4-} \cdots, \quad v=u-(1-c), \\
& =\frac{1}{\lambda} \int_{0}^{\infty} e^{-v t}\left[\frac{1-c}{e^{v(1-c)}-c}\right]^{2} d v \quad(\text { Wall }[17,(92.17)]),
\end{aligned}
$$

where $v=(z-\lambda+\mu) / \lambda$. Inverting this Laplace transform,

$$
L^{-1}(R(z))=\frac{e^{(\lambda-\mu) t}(\lambda-\mu)^{2}}{\left[\lambda e^{(\lambda-\mu) t}-\mu\right]^{2}} .
$$

This is a well-known result of a simple birth and death process (Bailey [2, page 94]). Thus

$$
P_{10}(t)=e^{-v t} \frac{e^{(\lambda-\mu) t}(\lambda-\mu)^{2}}{\left[\lambda e^{(\lambda-\mu) t}-\mu\right]^{2}} .
$$

We can find the time to tumour onset from (3.21):

$$
q_{0}(t) \longrightarrow \mu \int_{0}^{\infty} e^{-v t} \frac{e^{(\lambda-\mu) t}(\lambda-\mu)^{2}}{\left[\lambda e^{(\lambda-\mu) t}-\mu\right]^{2}} d t
$$


Example 4.2 (given in the previous section). Here $\lambda_{n}=\lambda, \mu_{n}=\mu$ :

$$
\begin{aligned}
R(z) & =\frac{1}{z+\lambda+\mu-} \frac{\lambda \mu}{z+\lambda+\mu-} \cdots \\
& =\frac{1}{z+\lambda+\mu-R(z) \lambda \mu} \lambda \mu(R(z))^{2}-(z+\lambda+\mu) R(z)+1=0, \\
R(z) & =\frac{z+\lambda+\mu \pm \sqrt{(z+\lambda+\mu)^{2}-4 \lambda \mu}}{2 \lambda \mu} \\
& =\frac{2 \lambda \mu}{z+\lambda+\mu+\sqrt{(z+\lambda+\mu)^{2}-4 \lambda \mu}}, \\
L^{-1}(R(z)) & =\frac{2 I_{1}(\alpha t) e^{-(\lambda+\mu) t}, \quad \alpha=2 \sqrt{\lambda \mu},}{\alpha t}, \quad\left(1-e^{-v(t-y)}\right) d y \\
q_{0}(t) & =e^{-v t+\mu \int_{0}^{t} \frac{2 I_{1}(\alpha y) e^{-(\lambda+\mu+\nu) y}}{\alpha y}} \quad \text { as } t \longrightarrow \infty .
\end{aligned}
$$

In the following examples, $\lambda_{n}+\mu_{n}=a_{n}$ and $\lambda_{n} \mu_{n+1}=b_{n}$ are known for $n=1,2,3, \ldots$ For an arbitrary $\mu_{1}>0$, find $\lambda_{1}$ from $\lambda_{1}+\mu_{1}, \mu_{2}$ from $\lambda_{1} \mu_{2}, \lambda_{2}$ from $\lambda_{2}+\mu_{2}, \mu_{3}$ from $\lambda_{2} \mu_{3}$, and so forth.

Specifically, for $n=1,2,3, \ldots$,

$$
\lambda_{n}=a_{n}-\mu_{n}, \quad \mu_{n+1}=b_{n} \frac{A_{n-1}}{B_{n}}
$$

where

$$
B_{n}=\left|\begin{array}{cccccc}
a_{n} & 1 & & & & \\
b_{n-1} & a_{n-1} & 1 & & \\
& b_{n-2} & a_{n-2} & 1 & & \\
& & & \ddots & & 1 \\
& & & & b_{1} & a_{1}-\mu_{1}
\end{array}\right|_{n \times n},
$$

and $A_{n-1}$ is obtained after deleting first row and first column of the determinant $B_{n}$ with $B_{1}=a_{1}-\mu_{1}$ and $A_{0}=1$.

We have taken specifically certain continued fractions which are continued fractions expansions of Laplace transforms of known functions. Such solutions are useful in gaining insights and for comparing the tumour onset with different density-dependent birth, 
death, and immigration parameters. We have concentrated on intermediate and tumour cells and have not taken into account the normal cells. However, the rate of normal cells becoming intermediate can be included along with the birth rate of the intermediate cells.

Example 4.3. Here, for $n=1,2,3, \ldots$,

$$
\begin{aligned}
\lambda_{n}+\mu_{n} & =a+2(n-1), \\
\lambda_{n} \mu_{n+1} & =n(n+a-1), \quad a>0, \\
R(z) & =\frac{1}{z+a-} \frac{a}{z+a+2-} \frac{2(a+1)}{z+a+4-} \ldots \\
& =\int_{0}^{\infty} \frac{e^{-z t}}{(1+t)^{a}} d t \quad \text { (Lorentzen and Waadeland [8, page 583]), } \\
L^{-1}(R(z)) & =\frac{1}{(1+t)^{a}}, \quad a>0 .
\end{aligned}
$$

From (3.15)

$$
P_{10}(t)=\frac{e^{-v t}}{(1+t)^{a}}, \quad a>0
$$

and $q_{0}(t)$ can be found from (3.21) and

$$
q_{0}(t) \longrightarrow \mu_{1} \int_{0}^{\infty} \frac{e^{-v t}}{(1+t)^{a}} d t
$$

Example 4.4. Let $\alpha>0,0<c<1$,

$$
\begin{aligned}
\lambda_{n}+\mu_{n} & =a+(n-1)(1+c), \\
\lambda_{n} \mu_{n+1} & =(a+n-1) n c, \quad n=1,2,3, \ldots, \\
R(z) & =\frac{1}{z+a-} \frac{a c}{z+c+a+1-} \cdots \\
& =\int_{0}^{\infty} e^{-z t} \frac{(1-c)^{a} e^{-a(1-c) t}}{\left[1-c e^{-(1-c) t}\right]^{a}} d t \quad(\text { Wall }[17,(92.17)]), \\
P_{10}(t) & =\frac{e^{-v t}(1-c)^{a}}{\left[1-c e^{-(1-c) t}\right]^{a} c}, \\
q_{0}(t) & \longrightarrow \int_{0}^{\infty} \frac{e^{-v t}(1-c)^{a}}{\left[1-c e^{-(1-c) t}\right]^{a}} \quad \text { as } t \longrightarrow \infty .
\end{aligned}
$$

Note that Example 4.2 is a special case of this example. 
2666 Density-dependent carcinogenesis models

Example 4.5. Here, for $n=1,2,3, \ldots$,

$$
\begin{aligned}
\lambda_{n}+\mu_{n} & =\frac{(N-1) \alpha}{2}, \\
\lambda_{n} \mu_{n+1} & =\frac{n^{2}\left(N^{2}-n^{2}\right)}{2^{2}\left(4 n^{2}-1\right)} \alpha^{2}, \quad n=1,2, \ldots, N-1, \\
R(z) & =\frac{1}{z+((N-1) / 2) \alpha-} \frac{1^{2} \alpha^{2}\left(N^{2}-1^{2}\right) / 2^{2}\left(4.1^{2}-1\right)}{z+((N-1) / 2) \alpha-} \ldots \\
& =\frac{1}{N} \sum_{j=0}^{N-1} \frac{1}{z+j} \quad(\text { Bowman and Shenton }[4, \text { page 29]), } \\
L^{-1}(R(z)) & =\frac{1}{N} \sum_{j=0}^{N-1} e^{-j \alpha t}, \\
P_{10}(t) & =\frac{1}{N} \sum_{j=0}^{N-1} e^{-(v+j \alpha) t}, \\
q_{0}(t) & \longrightarrow \mu_{1} \frac{1}{N} \sum_{j=0}^{N-1} \frac{1}{v+j} \quad \text { as } t \longrightarrow \infty .
\end{aligned}
$$

Example 4.6.

$$
\begin{aligned}
\lambda_{n}+\mu_{n} & =(N-n+1) p+(n-1) q, \quad n=1,2,3, \ldots, N-1, \\
\lambda_{n} \mu_{n+1} & =(N-n+1) n p q, \quad n=1,2, \ldots, N-1, \\
b(N, j, p)=\left(\begin{array}{c}
N \\
j
\end{array}\right) p^{j} q^{N-j}, \quad j=0,1,2, \ldots, N, & \quad(\text { Bowman and Shenton }[4, \text { page } 30]), \\
R(z)= & \frac{1}{z+N p-} \frac{N p q}{z+(N-1) p+q-} \cdots \frac{p q}{z+p+(N-1) q} \\
= & \sum_{j=0}^{N} \frac{b(N, j, p)}{z+j}, \\
L^{-1}(R(z))= & \left(q+p e^{-t}\right)^{N}, \\
P_{10}(t)= & e^{-v t}\left(q+p e^{-t}\right)^{N}, \\
q_{0}(t) & \longrightarrow \mu_{1} \sum_{j=0}^{N} \frac{b(N, j, p)}{v+j} .
\end{aligned}
$$

\section{Discussion}

In the literature, much attention has been paid to carcinogenesis models with constant birth, death, and mutation rates. But cancer assumes myriad forms. In this paper, we have discussed models with density-dependent rates. When these rates are known, one 
can numerically invert the Laplace transform of the time to tumour onset. We have given explicit expressions for this quantity in specific cases.

\section{Acknowledgment}

P. R. Parthasarathy thanks the Alexander von Humboldt Stiftung for financial assistance during the preparation of the paper.

\section{References}

[1] E. K. Afenya and C. P. Calderón, Diverse ideas on the growth kinetics of disseminated cancer cells, Bull. Math. Biol. 62 (2000), no. 3, 527-542.

[2] N. T. J. Bailey, The Elements of Stochastic Processes with Applications to the Natural Sciences, John Wiley \& Sons, New York, 1964.

[3] B. C. Berndt, Ramanujan's Notebooks. Part II, Springer, New York, 1989.

[4] K. O. Bowman and L. R. Shenton, Continued Fractions in Statistical Applications, Statistics: Textbooks and Monographs, vol. 103, Marcel Dekker, New York, 1989.

[5] J. Denes and D. Krewski, An exact representation for the generating function for the MoolgavkarVenzon-Knudson two-stage model of carcinogenesis with stochastic stem cell growth, Math. Biosci. 131 (1996), no. 2, 185-204.

[6] W. W. Gibbs, Untangling the roots of cancer, Sci. Amer. 289 (2003), no. 1, 57-65.

[7] W. B. Jones and W. J. Thron, Continued Fractions. Analytic Theory and Applications, Encyclopedia of Mathematics and Its Applications, vol. 11, Addison-Wesley, Massachusetts, 1980.

[8] L. Lorentzen and H. Waadeland, Continued Fractions with Applications, Studies in Computational Mathematics, vol. 3, North-Holland, Amsterdam, 1992.

[9] E. G. Luebeck and S. H. Moolgavkar, Multistage carcinogenesis and the incidence of colorectal cancer, Proc. Nat. Acad. Sci. U.S.A. 99 (2002), no. 23, 15095-15100.

[10] S. H. Moolgavkar, Scientific Issues in Quantitative Cancer Risk Assessment, Birkhäuser, Massachusetts, 1990.

[11] Stochastic models of carcinogenesis, Handbook of Statistics (C. R. Rao and R. Chakraborty, eds.), vol. 8, Elsevier, New York, 1991, pp. 373-393.

[12] S. H. Moolgavkar and E. G. Luebeck, Multistage carcinogenesis: population-based model for colon cancer, J. Natl. Cancer Inst. 84 (1992), no. 8, 610-618.

[13] S. H. Moolgavkar and D. J. Venzon, Two-event model for carcinogenesis: Incidence curves for childhood and adult tumors, Math. Biosci. 47 (1979), 55-77.

[14] J. A. Murphy and M. R. O'Donohoe, Some properties of continued fractions with applications in Markov processes, J. Inst. Math. Appl. 16 (1975), no. 1, 57-71.

[15] P. R. Parthasarathy and R. B. Lenin, Birth and Death Process (BDP) Models with ApplicationsQueueing, Communication Systems, Chemical Models, Biological Models: The State-of-theArt with a Time-Dependent Perspective, American Series in Mathematical and Management Sciences, vol. 51, American Science Press, Columbus, 2004.

[16] W. Y. Tan, Stochastic Models of Carcinogenesis, Marcel Dekker, New York, 1991.

[17] H. S. Wall, Analytic Theory of Continued Fractions, D. Van Nostrand, New York, 1948.

P. R. Parthasarathy: Department of Mathematics, Indian Institute of Technology, Madras, Chennai 600036, India

E-mail address: prp@iitm.ac.in

Klaus Dietz: Department of Medical Biometry, University of Tuebingen, 72070 Tuebingen, Germany

E-mail address: klaus.dietz@uni-tuebingen.de 


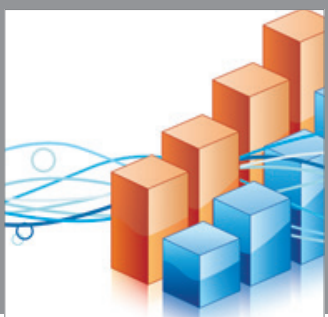

Advances in

Operations Research

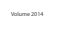

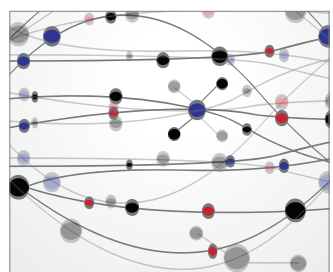

\section{The Scientific} World Journal
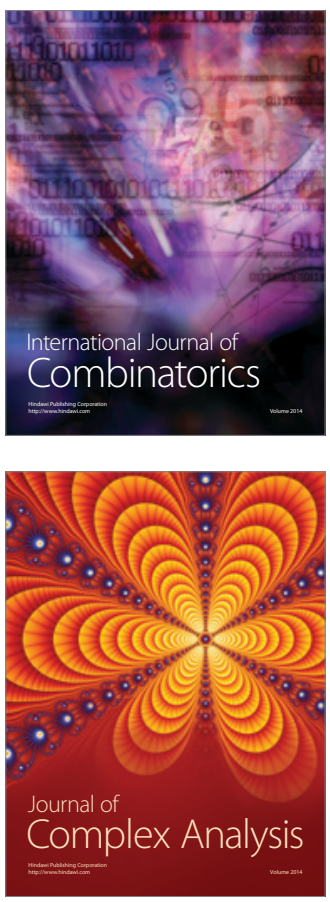

International Journal of

Mathematics and

Mathematical

Sciences
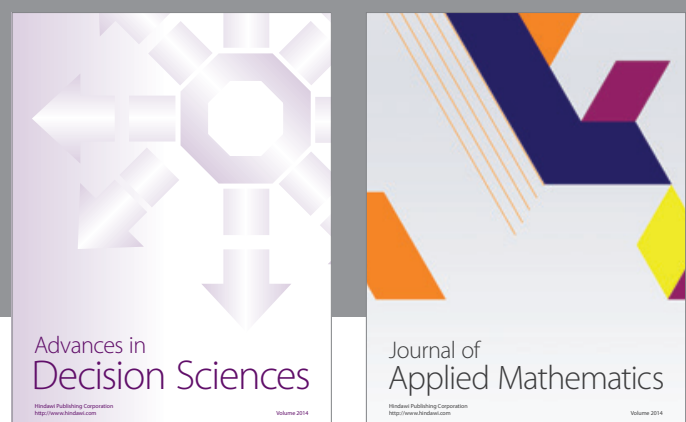

Journal of

Applied Mathematics
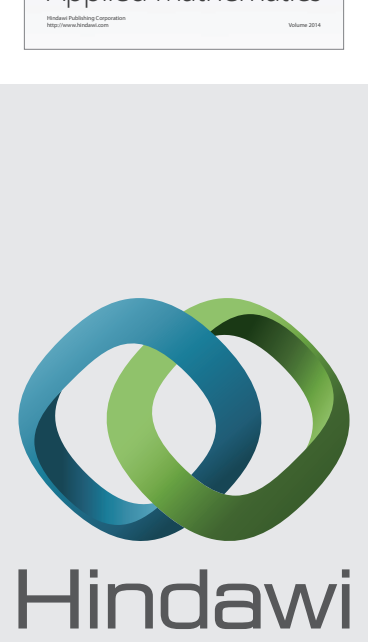

Submit your manuscripts at http://www.hindawi.com
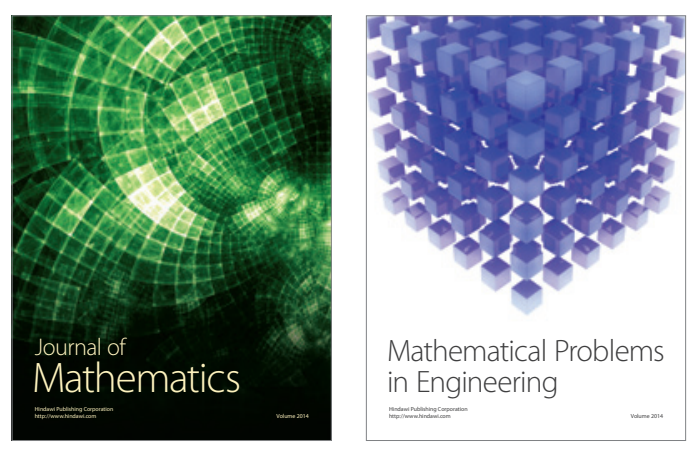

Mathematical Problems in Engineering
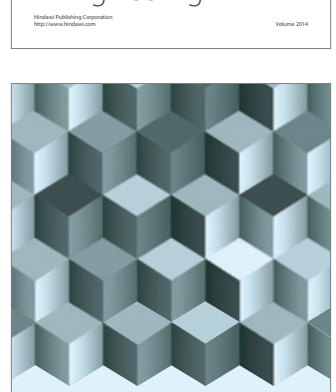

Journal of

Function Spaces
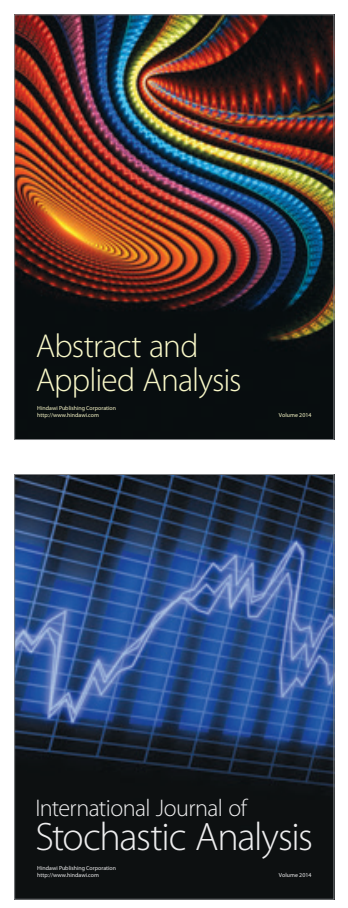

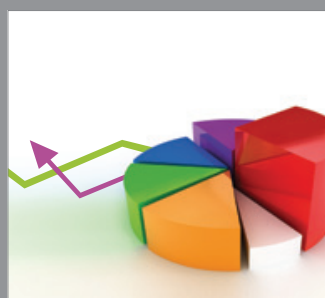

ournal of

Probability and Statistics

Promensencen
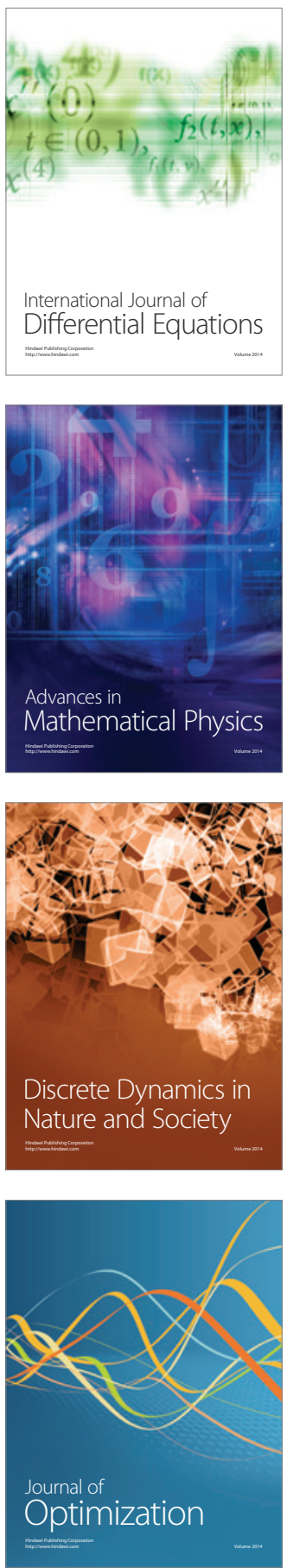